Supporting Information

\title{
Drug Penetration Analysis in 3D Cell Cultures Using Fiducial-Based Semi-automatic Coregistration of MALDI MSI and Immunofluorescence Images
}

\author{
Markéta Machálková ${ }^{1}$, Barbora Pavlatovská ${ }^{2}$ Jan Michálek ${ }^{3}$, Adam Pruška ${ }^{1}$, Karel Štěpka ${ }^{3}$, Tereza Nečasová ${ }^{3}$, \\ Katarzyna Anna Radaszkiewicz², Michal Kozubek² ${ }^{3}$ Jan Šmarda², Jan Preisler ${ }^{1}$, Jarmila Navrátilová*2,4 \\ ${ }^{1}$ Department of Chemistry, Faculty of Science and Central European Institute of Technology (CEITEC), \\ Masaryk University, Kamenice 5, 62500 Brno, Czech Republic \\ ${ }^{2}$ Department of Experimental Biology, Faculty of Science, Masaryk University, Kamenice 5, 62500 \\ Brno, Czech Republic \\ ${ }^{3}$ Centre for Biomedical Image Analysis, Faculty of Informatics, Masaryk University, Botanická 68a, 602 \\ 00 Brno, Czech Republic \\ ${ }^{4}$ Center for Biological and Cellular Engineering, International Clinical Research Center, St. Anne's \\ University Hospital, Pekařská 53, 65691 Brno, Czech Republic
}

*Corresponding author:

Jarmila Navrátilová

Contact information: 22031@ mail.muni.cz; +420549498912 
This supporting information includes the following:

The mixture of phosphatidylcholines (PC), drugs and peptide implemented for calibration of MALDI MSI

Sensitivity of the distance-dependent profiles to variations in registration parameters

Perifosine ionization suppression

MALDI MS from a spheroid induced by perifosine

The effect of MALDI MSI on subsequent IHC analysis

Descriptive statistics of the antibody abundances within the peels

Whole spheroid cross-sectioning

LCM and LC-ESI MS analysis of a spheroid section

Perifosine profile variability

The mixture of phosphatidylcholines (PC), drugs and peptide for calibration of MALDI MSI

The calibration mixture was prepared for the $m / z$ range $340-1000$ in the positive ionization mode.

All calibrants were detected as protonated molecules, and the cubic enhanced calibration mode was used (see Table S1).

Table S1: The calibration mixture for MALDI MSI

\begin{tabular}{|c|c|c|c|}
\hline & standard & {$[\mathbf{M}+\mathbf{H}]^{+}$} & concentration $[\boldsymbol{\mu M}]$ \\
\hline 1 & $06: 0$ Lyso PC & 356.183265 & 30 \\
\hline 2 & miltefosine & 408.323722 & 40 \\
\hline 3 & perifosine & 462.370672 & 25 \\
\hline 4 & $18: 0$ Lyso PC & 524.371066 & 50 \\
\hline 5 & Akt 1 2 inhibitor & 552.250635 & 750 \\
\hline 6 & DGEFR peptide & 623.278366 & 150 \\
\hline 7 & $16: 0$ PC & 734.569432 & 50 \\
\hline 8 & $16: 0-18: 1$ PC & 760.585082 & 100 \\
\hline 9 & $18: 0$ PC & 790.632032 & 400 \\
\hline 10 & $20: 0$ PC & 846.694632 & 400 \\
\hline
\end{tabular}




\section{Sensitivity of the distance-dependent profiles to variations in registration parameters}

The only user-selectable parameters in the whole process of creating the distance-dependent mass or antibody profiles in the peels are the thresholds used for the extraction of fiducial markers from either the MALDI MSI fiducial $\mathrm{m} / \mathrm{z}$ window or the LSCM transmission bright-field scan. To determine how sensitive the distance-dependent profiles were to variations in threshold levels, we carried out numerical sensitivity analysis in which we calculated what percentage change in the distance-dependent perifosine or IHC antibody profile was caused by some percentage change in a threshold value. Since registration accuracy is affected by the choice of the threshold values, sensitivity defined as the ratio of the above percent changes evaluates how strongly the distance-dependent profiles depend on the registration accuracy.

We defined the threshold percentage change as

$\Delta \vartheta=100 \cdot\left(\vartheta-\vartheta_{r e f}\right) / \vartheta_{r e f}[\%]$

where $\vartheta_{r e f}$.default threshold value for MALDI MSI perifosine ion count or LSCM fluorescence signal

$\vartheta \quad$..perturbed threshold value for MALDI MSI perifosine ion count or LSCM fluorescence signal

Our definition of the profile percentage change relies on the $\mathrm{L}^{1}$ norm of a sequence.

The $\mathrm{L}^{1}$ norm of the reference profile is

$\left\|\rho_{\text {ref }}\right\|_{1}=\sum_{p=1}^{N}\left|\rho_{r e f, p}\right|$

where $\rho_{\text {ref }} \quad$...reference mean perifosine ion count profile or reference mean fluorescence signal profile

$\rho_{\text {ref,p }} .$. value of $\rho_{\text {ref }}$ at the peel $p$

$N \quad$.. number of peels

and the $\mathrm{L}^{1}$ norm of the profile change caused by some perturbation of a threshold value reads 
$\|\Delta \rho\|_{1}=\sum_{p=1}^{N}\left|\rho_{p}-\rho_{\text {ref }, p}\right|$

where $\rho_{r e f, p}$. reference mean perifosine ion count

or reference mean fluorescence signal at the peel $p$

$\rho_{p} \quad$.. perturbed mean perifosine ion count

or perturbed mean fluorescence signal at the peel $p$

$N \quad$.. number of peels

We defined the percentage change in the distance-dependent profile (perifosine or IHC antibody) as

$\Delta \bar{\rho}=\frac{\|\Delta \rho\|_{1}}{\left\|\rho_{\text {ref }}\right\|_{1}} \cdot 100[\%]$

and the sensitivity $\sigma$ as

$\sigma=\frac{\Delta \bar{\rho}}{\Delta \vartheta}$

We varied the LSCM threshold percentage change between $-50 \%$ and $50 \%$ around the value of 50000 while keeping the threshold for MALDI MSI fiducials constant at 0.09 and obtained sensitivity values in Table S2:

Table S2: Sensitivity values with constant MALDI MSI fiducial threshold

\begin{tabular}{|c|c|c|c|c|}
\hline $\begin{array}{c}\text { LSCM threshold } \\
\text { percentage change }\end{array}$ & $-50 \%$ & $-25 \%$ & $25 \%$ & $50 \%$ \\
\hline LSCM sensitivity & -0.26 & -0.55 & 0.024 & 0.035 \\
\hline MALDI sensitivity & -0.31 & -0.74 & 0.046 & 0.022 \\
\hline
\end{tabular}

For example, the sensitivity value of 0.022 in the lower right corner of the above table tells us that if the LSCM threshold is increased by $50 \%$, the distance-dependent profile increases by $1 \%$. This is a very low sensitivity of the profile to the parameter variation, as desired.

Similarly, keeping the LSCM threshold constant at 50000, the MALDI MSI threshold percentage change varied by approximately 0.09 between $-50 \%$ and $50 \%$, yielding the sensitivity values shown in Table S3. 
Table S3: Sensitivity values with a constant LSCM fiducial threshold

\begin{tabular}{|c|c|c|c|c|}
\hline $\begin{array}{l}\text { MALDI threshold } \\
\text { percentage change }\end{array}$ & $-50 \%$ & $-25 \%$ & $25 \%$ & $50 \%$ \\
\hline LSCM sensitivity & -0.29 & -0.45 & 0.14 & 0.059 \\
\hline MALDI sensitivity & -0.35 & -0.62 & 0.047 & 0.083 \\
\hline
\end{tabular}

The sensitivity values we observed show that even massive deviations from manually selected threshold settings are always attenuated in the resulting profile percentage change. For negative threshold perturbations of both LSCM and MALDI MSI fiducial thresholds, profile changes are almost an order of magnitude larger than for positive parameter changes. This indicates that the manually selected parameters are close to the lower bound of the insensitivity range and that the peeling procedure is robust with respect to unoptimized parameters. 


\section{Perifosine ionization suppression}

A solution of $10 \mu \mathrm{M}$ perifosine in $50 \% \mathrm{EtOH}$ was sprayed by iMatrixSpray (Tardo $\mathrm{GmbH}$, Switzerland) over a section of perifosine-untreated spheroid in gelatin. The parameters of spraying were as follows: speed of spraying nozzle, $180 \mathrm{~mm} / \mathrm{s}$; height of nozzle, $70 \mathrm{~mm}$; line distance, $1 \mathrm{~mm}$; density, $2.38 \mu \mathrm{L} / \mathrm{cm}^{2}$; and number of cycles, 4 . The samples were allowed to dry and were sublimed by DHB matrix, and MALDI MSI analysis was undertaken with standard settings (see Experimental section). The ion intensities at $\mathrm{m} / \mathrm{z}, 462.45$ in the spheroid and gelatin areas were exported from FlexImaging 5.0 software to FlexAnalysis 3.4. The average values, standard deviations and the limit of detection (LOD) of perifosine in the spheroid were calculated in MS Excel. The perifosine signal map across the sample is depicted in Figure S1. Furthermore, another spheroid was treated with $20 \mu \mathrm{M}$ perifosine in cultivation medium. After $24 \mathrm{~h}$, the spheroid was prepared for MALDI MSI analysis as usual and processed by peeling algorithm (using the coregistration with TO-PRO to precisely determine the spheroid boundaries). The average perifosine abundances in peels are depicted in the graph in Figure S2.
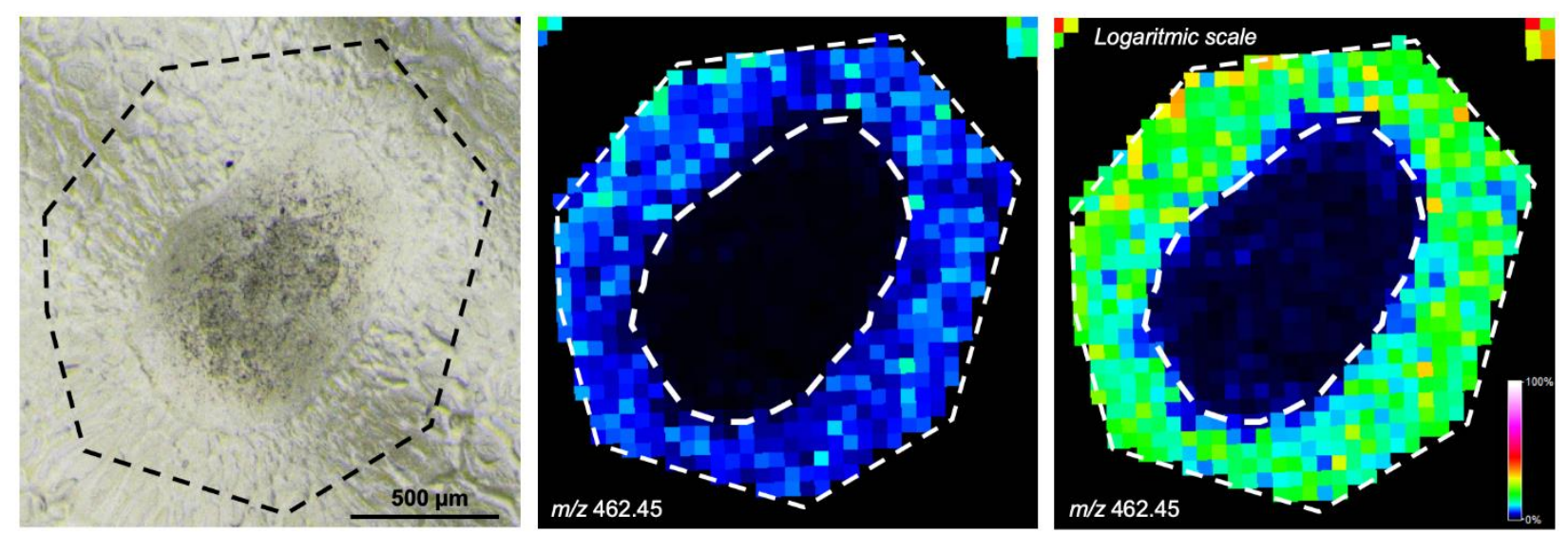

Figure S1: Optical and MALDI MS images of perifosine sprayed over the section of perifosine-untreated spheroid and surrounding gelatin. The perifosine level was 2.4 fmol per pixel. 


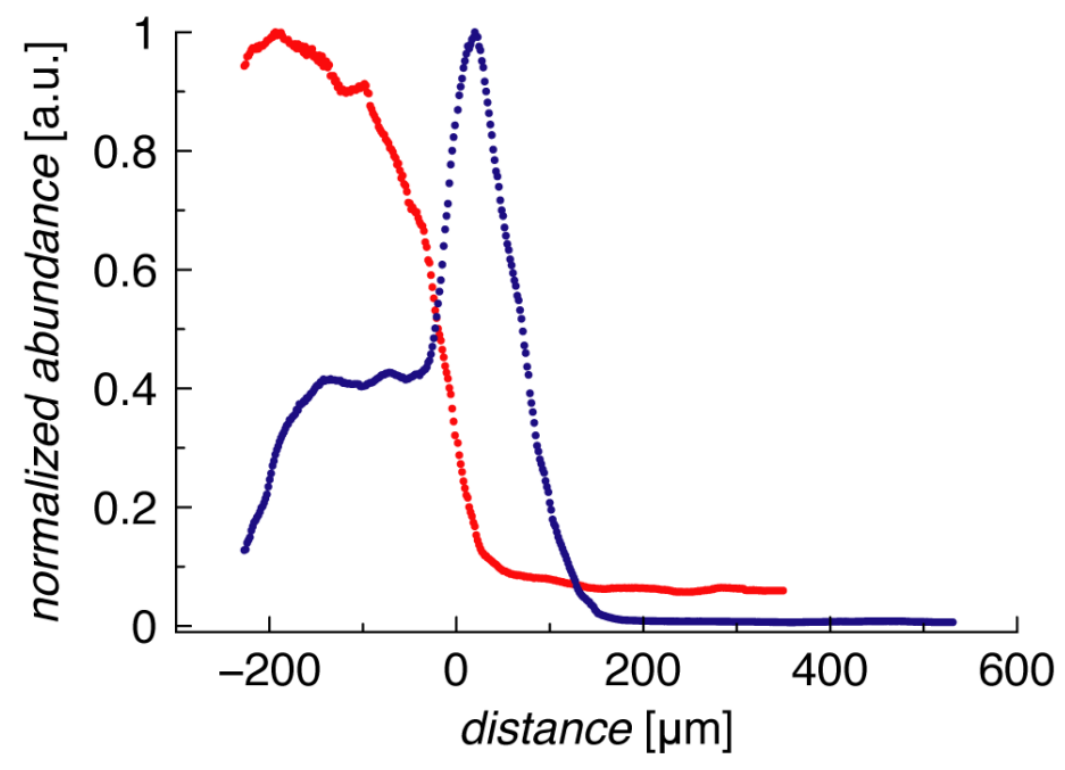

Figure S2: Perifosine abundance across the peels outside $(-230-0 \mu \mathrm{m})$ and inside $(0-550 \mu \mathrm{m})$ a spheroid section sprayed with perifosine (red trace) or a section from a spheroid treated by perifosine for $24 \mathrm{~h}$ (blue trace). The zero value on the $x$ axis represents the spheroid boundary. 


\section{MALDI MS from a spheroid induced by perifosine}

A representative mass spectrum (Figure S3) was obtained with the standard MALDI MSI settings from a pixel selected from outer spheroid cells to which perifosine penetrated after $24 \mathrm{~h}$ induction.

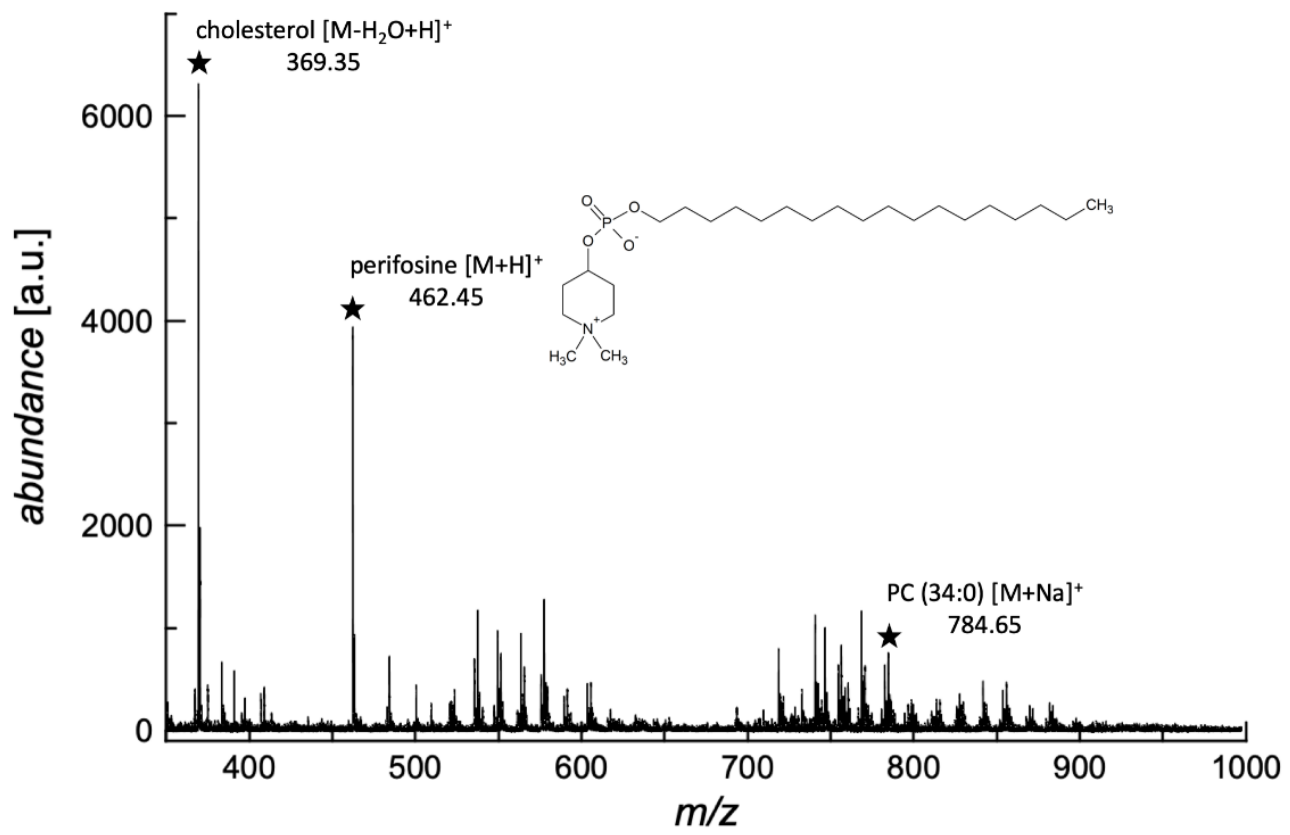

Figure S3: A MALDI mass spectrum of a spheroid induced by perifosine with DHB matrix in positive ionization mode. 


\section{The effect of MALDI MSI on subsequent fluorescent IHC analysis}

The spheroid sections were covered with MALDI matrix, subjected to MALDI MSI analysis, washed and followed by fluorescent IHC analysis. Alternatively, they were treated according to the simple IHC protocol. Individual burnt pixels after MALDI MSI are shown in the second column in Figure $\mathrm{S} 4$, and the $\mathrm{Ab}$ and TO-PRO distributions in either MALDI-IHC or simple IHC are shown in the right part of the figure.

\begin{tabular}{|c|c|c|c|c|c|c|}
\hline & $\begin{array}{c}\text { Optical photo } \\
\text { prior to MALDI MSI }\end{array}$ & $\begin{array}{l}\text { Optical photo } \\
\text { after MALDI MSI }\end{array}$ & $\begin{array}{l}\text { MALDI IHC } \\
\qquad A b\end{array}$ & $\begin{array}{r}\mathrm{IHC} \\
\mathrm{Ab}\end{array}$ & $\begin{array}{c}\text { MALDI IHC } \\
\text { TO-PRO }\end{array}$ & $\begin{array}{c}\text { IHC } \\
\text { TO-PRO }\end{array}$ \\
\hline Ki67 & & & & & & \\
\hline $\begin{array}{l}\text { Cleaved } \\
\text { Caspase } \\
8\end{array}$ & & & & & & \\
\hline $\begin{array}{l}\text { SNAIL/ } \\
\text { SLUG }\end{array}$ & & & & & & \\
\hline
\end{tabular}

Figure S4: The influence of MALDI MSI on subsequent fluorescent IHC using three selected antibodies (red) and a cell nuclei marker (blue). 


\section{Descriptive statistics of the antibody abundances within the peels}
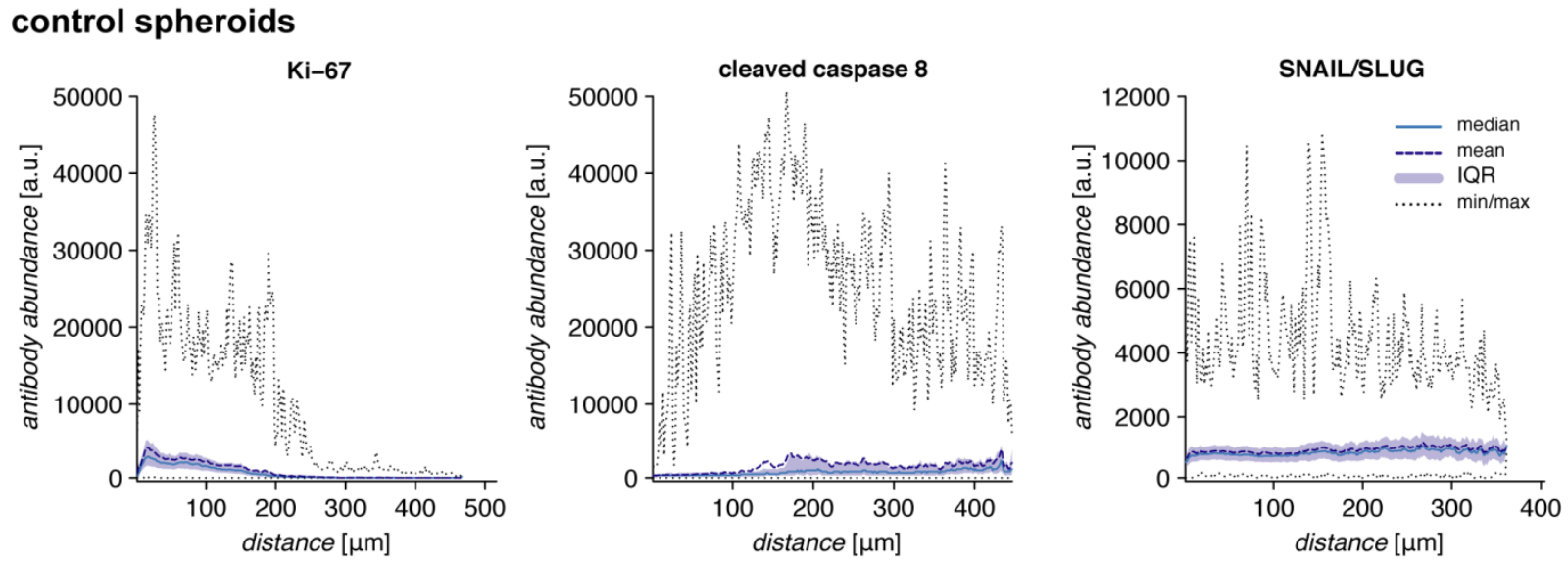

perifosine-treated spheroids
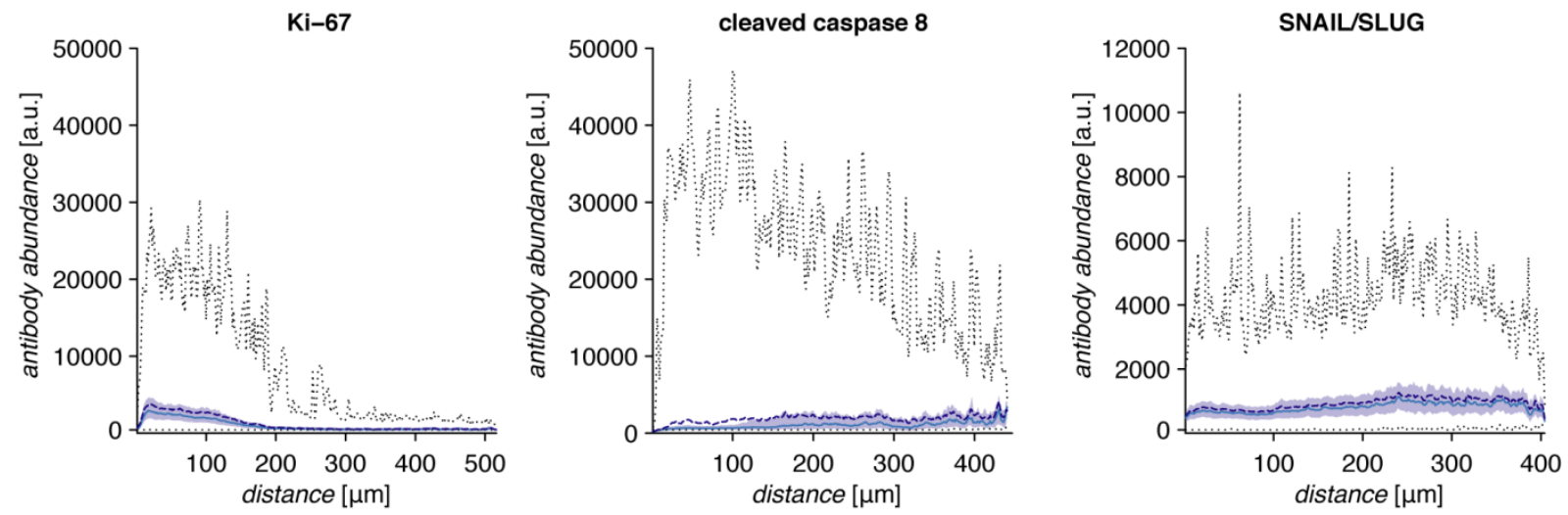

Figure S5: Descriptive statistics of antibody abundances in the control spheroids (first row) and the perifosine-treated spheroids (second row). The median, mean, minimum and maximum values of the detected signal are reported for each peel. The area of interquartile range (IQR, i.e., values between the 25 th and 75 th quantiles) is highlighted in violet. 


\section{Whole spheroid cross-sectioning}

A spheroid induced by perifosine for $24 \mathrm{~h}$ was prepared according to the MALDI MSI protocol and cross-sectioned along the Z-axis. Thirty sections were distributed to 3 glass slides, photographed with stereomicroscope zoom and coated with sublimed DHB. Perifosine distribution in 5 out of 8 MALDI MSI-imaged sections is shown in Figure S6.
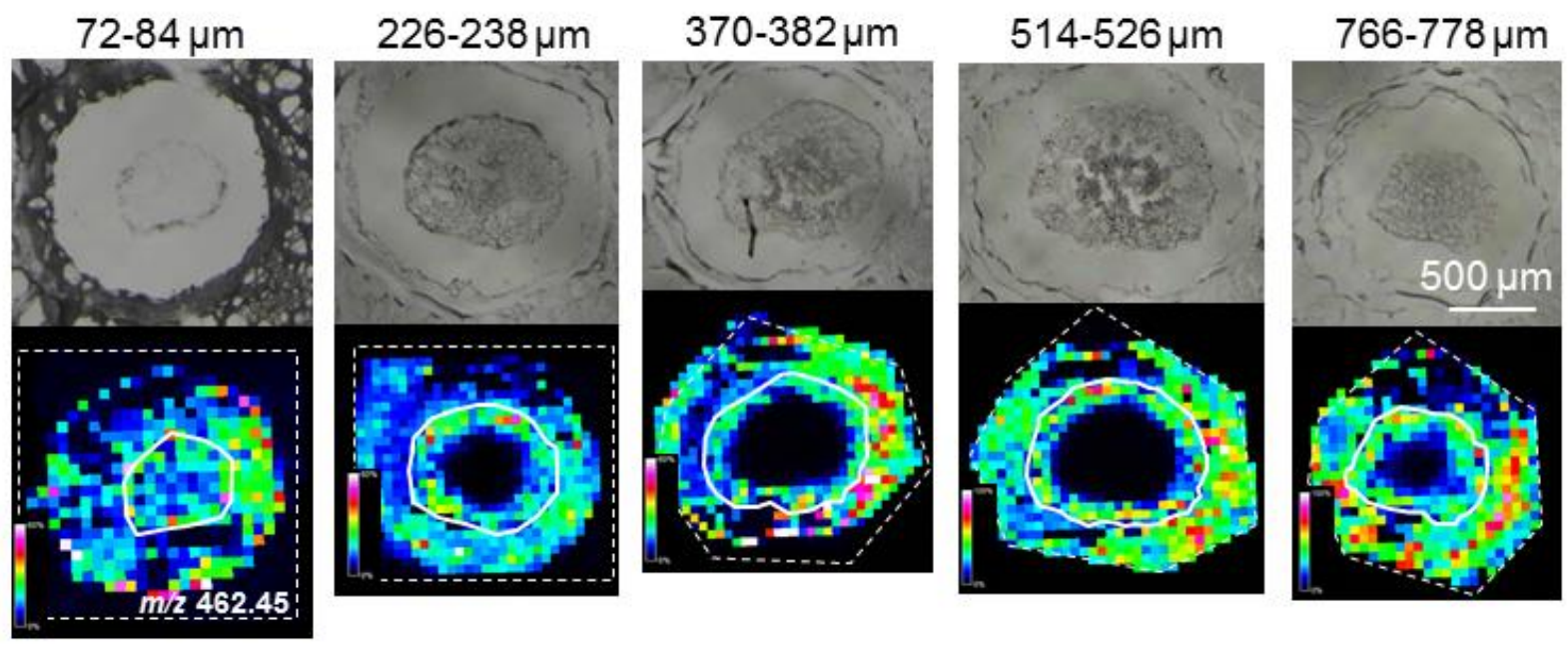

Figure S6: Photos and MALDI MS perifosine distributions in selected sections of a spheroid induced by perifosine for $24 \mathrm{~h}$. The positions of spheroid sections along the Z-axis are counted from the spheroid top $(0 \mu \mathrm{m})$. Dashed lines represent MALDI MS imaged areas, full lines represent spheroid tissue. 


\section{LCM and LC-ESI MS analysis of a spheroid section}

A spheroid was treated with perifosine for $24 \mathrm{~h}$. After that, the spheroid was cut into $25 \mu \mathrm{m}$ thick sections. The equatorial section was placed on a polyethylene naphthalate membrane slide (PEN, Carl Zeiss Ltd., Germany) for laser capture microdissection (LCM). LCM was conducted using a microdissection instrument (PALM MicroBeam, Carl Zeiss Ltd.) with a $355 \mathrm{~nm}$ laser. The conditions for LCM were optimized separately for spheroid and gelatin dissection. The spheroid section as well as the number and size of the ablated regions are pictured in Figure S7. The microdissected regions were placed in $7.5 \mu \mathrm{L} 50 \% \mathrm{EtOH}$ in a given microvial cap (30 spots from a particular region in each of the vial caps) and frozen until analysis. Perifosine was extracted in $10 \mu \mathrm{L} \mathrm{50 \%} \mathrm{EtOH,} \mathrm{and} \mathrm{the} \mathrm{samples} \mathrm{were} \mathrm{diluted} 3 \times$ by distilled water for LC-ESI MS (1200 LC system, 6224 TOF LC-MS; Agilent Technologies, CA, USA). The conditions for LC-ESI MS were derived from ${ }^{1,2}$ and are summarized in Table S4. The extracted ion chromatograms (EIC) of perifosine in the analyzed samples and their estimated quantities are depicted in Figure S8 and Table S5. A MALDI MS image of a $12 \mu \mathrm{m}$ thick equatorial section from the same spheroid is shown in Figure S9.

Table S4: LC-ESI MS conditions for perifosine detection

\begin{tabular}{|c|c|}
\hline LC stationary phase & Phenomenex Gemini NX-C18, $150 \times 4.6 \mathrm{~mm}, 3 \mu \mathrm{m}$ \\
\hline LC mobile phase & A: $95 \% 9 \mathrm{mM}$ ammonium formate in acetonitrile \\
\cline { 2 - 3 } & B: $95 \%$ acetonitrile, $5 \% 9 \mathrm{mM}$ ammonium formate \\
\hline LC mode & isocratic elution, A:B $40: 60(v / v)$ \\
\hline LC settings & flow rate: $0.8 \mathrm{~mL} / \mathrm{min}$, injection: $3 \mu \mathrm{L}$, analysis time: $10 \mathrm{~min}$ \\
\hline MS settings & $\begin{array}{c}\text { ESI+, nitrogen flow: } 7 \mathrm{~L} / \mathrm{min}, \text { gas temperature: } 350{ }^{\circ} \mathrm{C}, \\
\text { nebulizer: } 40 \mathrm{psig}, \text { fragmentor voltage: } 150 \mathrm{~V}, \text { capillary } \\
\text { voltage: }-3000 \mathrm{~V},[\mathrm{M}+\mathrm{H}]^{+}=462.3707, \text { EIC: } \mathrm{m} / \mathrm{z} 462.3700- \\
462.3730\end{array}$ \\
\hline
\end{tabular}




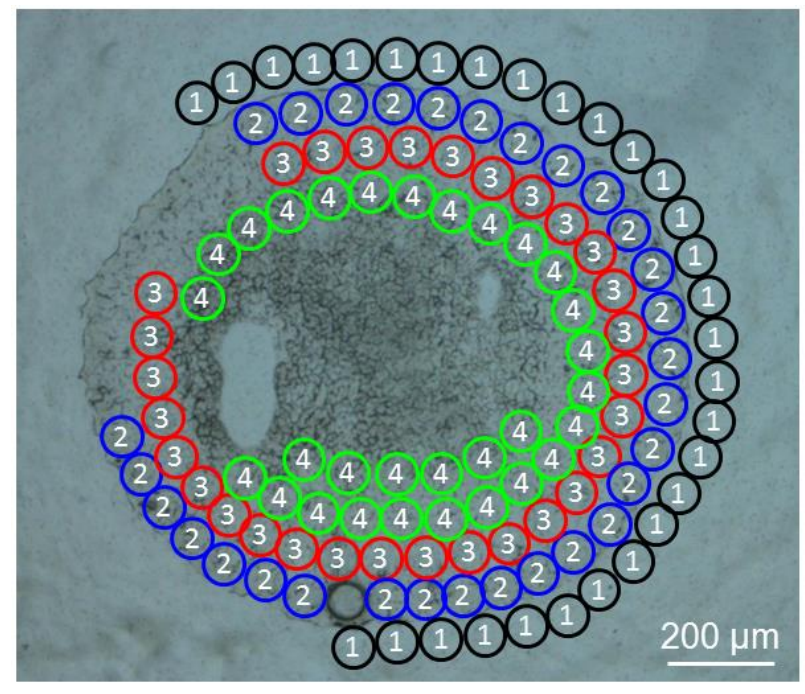

Figure S7: Photo of a gelatin-embedded spheroid section with microdissected regions (1 - gelatin, 2 boundary spheroid layer, 3 - middle spheroid layer, 4 - inner spheroid layer).

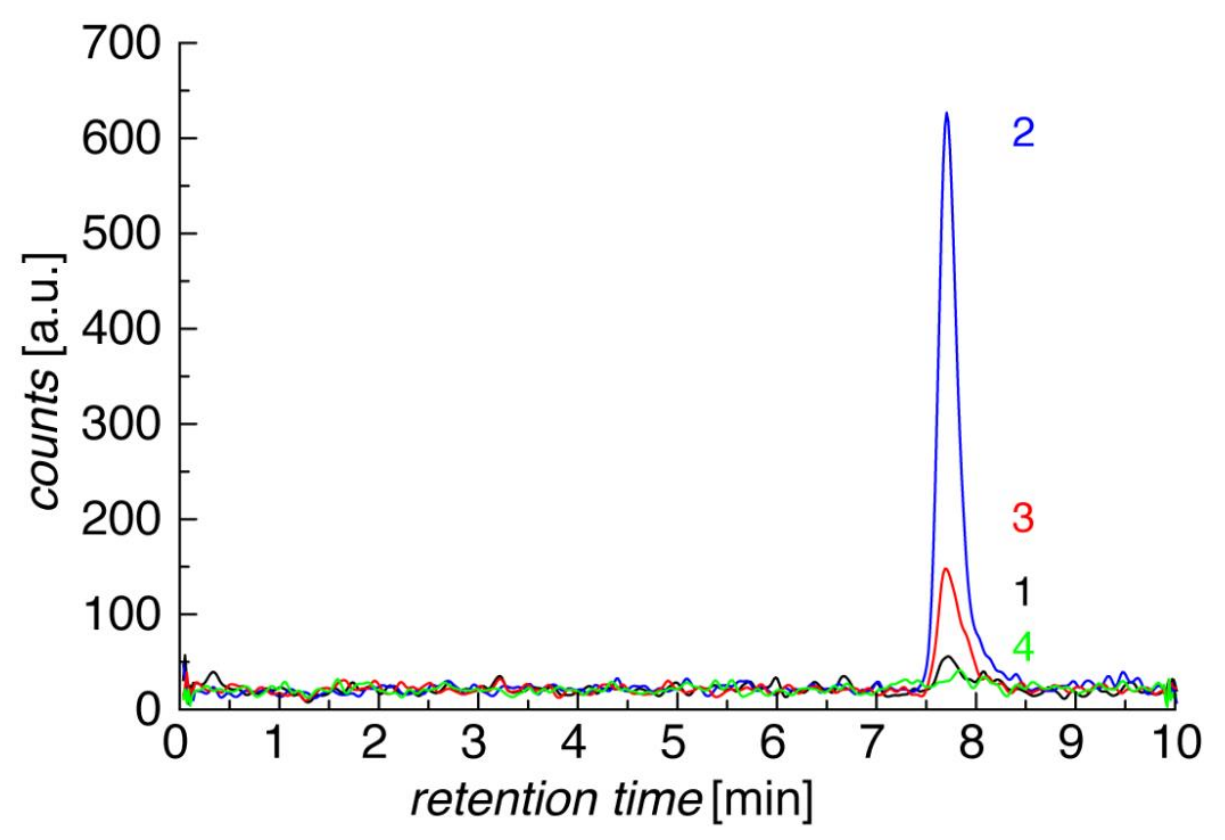

Figure S8: Extracted ion chromatograms (EIC) of perifosine in samples: 1 - gelatin, 2 - boundary spheroid layer, 3 - middle spheroid layer, 4 - inner spheroid layer. 
Table S5: Estimated quantity of perifosine in samples 1-4

\begin{tabular}{|c|c|c|c|}
\hline Sample & Region & $\begin{array}{c}\text { Distance from spheroid } \\
\text { surface }[\boldsymbol{\mu m}]\end{array}$ & $\begin{array}{c}\mathbf{c}[\mathbf{m M}] \text { in } \mathbf{1 0} \boldsymbol{\mu L} \text { of } \\
\text { sample }\end{array}$ \\
\hline $\mathbf{1}$ & gelatin & $-75-0$ & 6 \\
\hline $\mathbf{2}$ & boundary spheroid layer & $0-75$ & 921 \\
\hline $\mathbf{3}$ & middle spheroid layer & $75-150$ & 204 \\
\hline 4 & inner spheroid layer & $150-300$ & under LOD \\
\hline
\end{tabular}
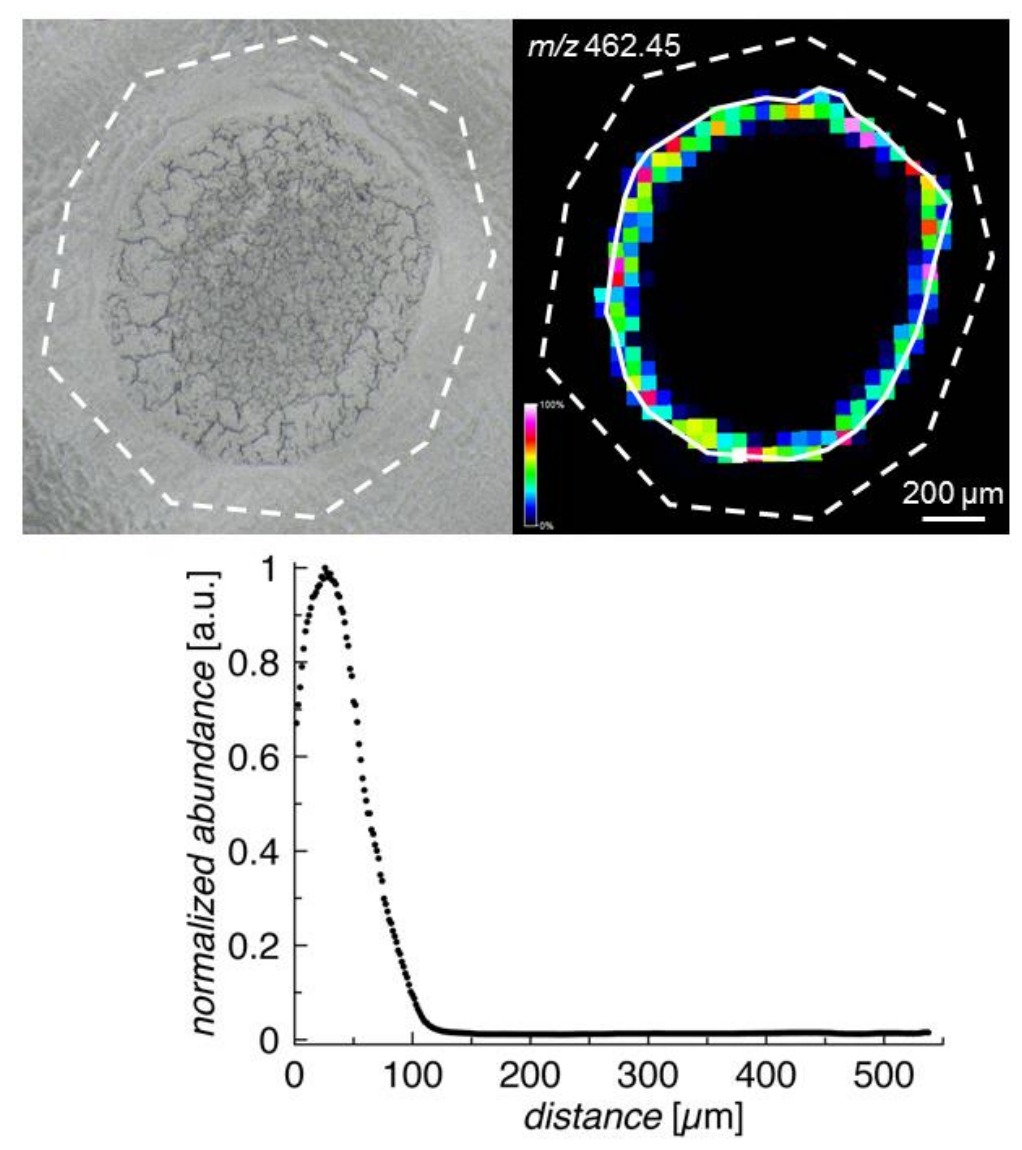

Figure S9: Photo and MALDI MS perifosine image of a spheroid treated by perifosine for $24 \mathrm{~h}$ (top) with the average perifosine abundance profile (bottom). 


\section{Perifosine profile variability}

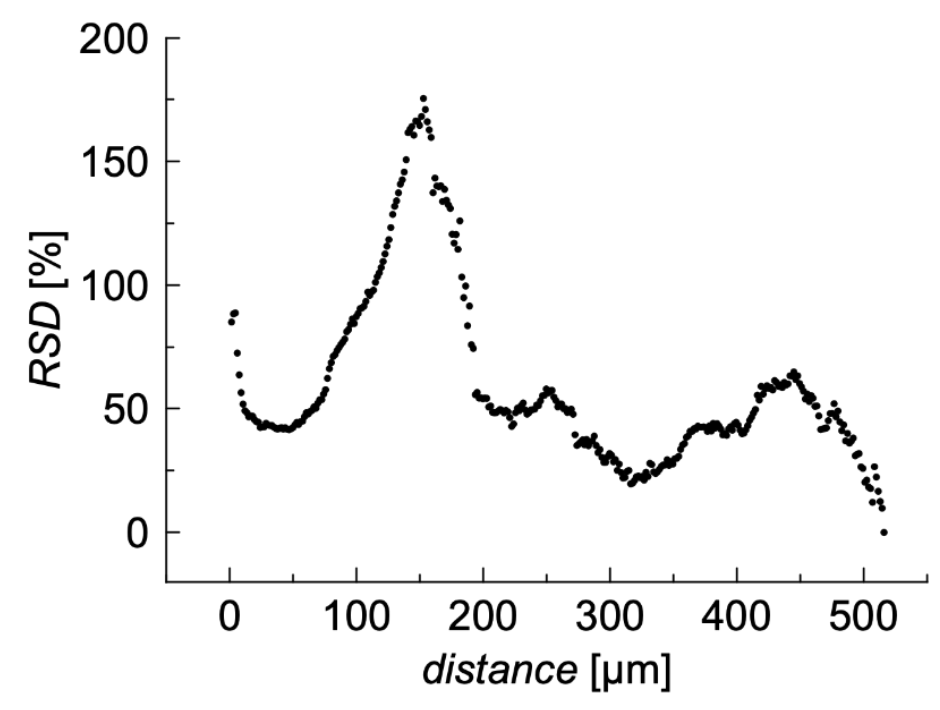

Figure S10: Relative standard deviation (RSD) of the perifosine signal in individual peels of a spheroid induced by perifosine (see the related graph in Figure 3D). The zero value on the $x$ axis represents the spheroid boundary. 


\section{References}

1. Woo, E.; Messmann, R.; Sausville, E.; Figg, W. Quantitative Determination of Perifosine, A Novel Alkylphosphocholine Anticancer Agent, in Human Plasma by Reversed-Phase Liquid Chromatography-Electrospray Mass Spectrometry. J Chrom B 2001, 759, 247-257, DOI: 10.1016/S0378-4347(01)00231-6.

2. Knebel, N.; Grieb, S.; Winkler, M.; Locher, M.; van der Vlis, E.; Verheij, E. Quantification of Perifosine, an Alkylphosphocholine Anti-Tumour Agent, in Plasma by Pneumatically Assisted Electrospray Tandem Mass Spectrometry Coupled with High-Performance Liquid Chromatography. J Chrom B 1999, 721, 257-269, DOI: 10.1016/S0378-4347(98)00469-1. 